\title{
George W. Wetherill (1925-2006): physicist, geochemist, planetary scientist, astrobiologist
}

\author{
Alan P. Boss \\ Carnegie Institution, Washington, DC, USA \\ email: boss@dtm.ciw.edu
}

\begin{abstract}
Trained as a physicist, George W. Wetherill (1925-2006) made seminal contributions to the fields of geochemical dating, meteoritical and asteroidal science, and the theory of the formation of terrestrial planets, evolving along the way into one of the first astrobiologists.
\end{abstract}

\section{Introduction}

George W. Wetherill was born on 12 August 1925 in Philadelphia, Pennsylvania. He attended the University of Chicago, earning four degrees: Ph.B., S.B., S.M., and Ph.D. Upon completion of his doctorate in nuclear physics (Wetherill 1953), Wetherill became a staff member at the Carnegie Institution's Department of Terrestrial Magnetism (DTM) in Washington, D.C., where physicists had branched out into new areas of science. In 1960, he left DTM to become a professor of geophysics and geology at UCLA, also serving as chairman of the Department of Planetary and Space Science from 1968 to 1972. In 1975, Wetherill returned to DTM as Director, a position he held until 1991. He remained on the active DTM staff until retiring in 2001. George Wetherill died on 19 July 2006.

\section{Research accomplishments}

Wetherill can be rightfully called the father of modern theories of the formation of the Earth. Wetherill was in the vanguard of the effort to place planet formation theory on a solid basis, but his work spanned much of the disciplines of Earth and planetary science.

Wetherill and his colleagues at DTM and Carnegie's Geophysical Laboratory revolutionized the field of geochemical dating of rocks. Wetherill conceived of the concordia diagram (Wetherill 1956, 1963), which uses the decay of radioactive uranium into lead to provide accurate dates for when the rocks crystallized. Wetherill's concordia diagram was a concept that found immediate and lasting acceptance, and stands as a singular achievement in the Earth sciences. It opened up the field of geological dating for events that happened billions of years ago on the Earth and on other rocky bodies (Wetherill 1975a).

Wetherill's second major contribution dealt with the orbital evolution of asteroids and of other small bodies in the Solar System (Wetherill 1967, 1969, 1974, 1987). He was the first to show that debris kicked out from meteorite impacts on Mars could be expected to end up on Earth, as has been spectacularly verified by the dozens of Martian meteorites found to date on Earth in Antarctica and elsewhere.

Using these skills in orbital dynamics, Wetherill launched into his third major research area, planet formation modeling, in 1975. He quickly became the world's leading authority on the process by which the rocky inner planets formed through impacts between progressively larger and larger bodies (Wetherill 1975b, 1976, 1980, 1990, 1994b). 
Wetherill was the first to point out that the Earth's formation involved impacts by bodies as large as Mars on the growing Earth (Wetherill 1985, 1986). One such giant impact is the now-accepted explanation for how the Earth's Moon was formed - an off-center giant impact produced a spray of hot rock that ended up in orbit around the Earth and then formed the Moon. In 1995, the first solid evidence of planetary systems orbiting other stars like our Sun was presented. Astronomers have since discovered over 350 planets in orbit around nearby stars, and recently may have found the first rocky planets. George Wetherill was way ahead of these discoveries (Wetherill 1991, 1994a, 1996). His models of terrestrial planet formation, combined with the latest discoveries, imply a rich future for those who seek to find Earth-like planets in our neighborhood of the galaxy.

\section{Honors}

Wetherill received a wide range of awards for his accomplishments throughout his life. He was a member of the U. S. National Academy of Sciences, a Fellow of the American Academy of Arts and Sciences, the American Geophysical Union, and the Meteoritical Society. He won the Harry H. Hess Medal of the American Geophysical Union, the Leonard Medal of the Meteoritical Society, the G. K. Gilbert Award of the Geological Society of America, the G. P. Kuiper Award of the Division of Planetary Sciences of the American Astronomical Society, and the Henry Norris Russell Lectureship of the American Astronomical Society. In 1997 Wetherill received the National Medal of Science, the nation's highest scientific award.

\section{Acknowledgements}

I thank the American Astronomical Society for a travel grant that partially supported my attendance at the Rio de Janeiro IAU General Assembly, as well as partial support from NASA Astrobiology Institute grant NCC2-1056.

\section{References}

Wetherill, G. W. 1953, Phys. Rev. 92, 907

Wetherill, G. W. 1956, I. Trans. Amer. Geophys. Union 37, 320

Wetherill, G. W. 1963, J. Geophys. Res. 68, 2957

Wetherill, G. W. 1967, J. Geophys. Res. 72, 2429

Wetherill, G. W. 1969, in: P. M. Millman (ed.), Meteorite Research (Dordrecht: D. Reidel), p. 579

Wetherill, G. W. 1974, Annual Rev. Earth Planet. Sci. 2, 303

Wetherill, G. W. 1975a, Ann. Rev. Nucl. Sci. 25, 283

Wetherill, G. W. 1975b, Proc. 6th Lunar Sci. Conf. (New York: Pergamon), p. 1539

Wetherill, G. W. 1976, Proceedings 7th Lunar Sci. Conf. (New York: Pergamon), p. 3245

Wetherill, G. W. 1980, Annual Review of Astronomy and Astrophysics 18, 77

Wetherill, G. W. 1985, Science 228, 877

Wetherill, G. W. 1986, in: W. K. Hartmann, R. J. Phillips \& G. J.Taylor (eds.), Origin of the Moon (Houston: Lunar and Planetary Institute), p. 519

Wetherill, G. W. 1987, Phil. Trans. R. Soc. Lond., Series A 323, 323

Wetherill, G. W. 1990, Ann. Rev. Earth. Plan. Sci. 18, 205

Wetherill, G. W. 1991, Science 253, 535

Wetherill, G. W. 1994a, Astrophysics \& Space Science 212, 23

Wetherill, G. W. 1994b, Geochimica et Cosmochimica Acta 58, 4513

Wetherill, G. W. 1996, Icarus 119, 219 